\title{
Erfahrungs-, (Kunst-)Werk- und Vermittlungsorientierung
}

\author{
Konkrete Zugänge zum medien- und kulturpädagogischen \\ Projektunterricht im empirischen Vergleich
}

\author{
Iwan Pasuchin ${ }^{1}$ (D) \\ ${ }^{1}$ Universität Mozarteum Salzburg
}

\begin{abstract}
Zusammenfassung
Sowohl innerhalb der handlungsorientierten Medienpädagogik als auch im Feld der Kulturellen Bildung wird die zentrale Stellung der Projektmethode hervorgehoben. Jedoch sind weder die Beweggründe zur Anwendung dieses Verfahrens noch dessen Alleinstellungsmerkmale eindeutig expliziert. Mit der Intention, Vermittlerinnen und Vermittler im medien- und kulturpädagogischen Bereich bei der Konzeption ihrer Interventionen zu unterstützen, wird im vorliegenden Beitrag der Forschungsfrage nachgegangen, mit welchen konkreten Zugängen zum Projektunterricht sich welche Ziele der Arbeit in diesem Spannungsfeld am ehesten erreichen lassen. Um das zu beantworten, erfolgt die Darstellung und Analyse von zehn wissenschaftlich begleiteten Workshops, die sich über mehrere Wochen, oft auch Monate erstreckten sowie grösstenteils von Medienkünstlerinnen und -künstlern an einer städtischen Haupt- bzw. Mittelschule durchgeführt wurden. Im Zuge dessen werden drei Herangehensweisen zur Arbeit an den Schnittstellen zwischen der Medienpädagogik und der Kulturellen Bildung extrahiert und näher beschrieben: die Erfahrungsorientierung, die Ausrichtung am (Kunst-)Werk sowie die Fokussierung auf Vermittlungsaspekte. Abschliessend findet der Vergleich dieser Ansätze hinsichtlich der Forschungsfrage statt, was im Aufzeigen einiger praktischer Konsequenzen mündet.
\end{abstract}

Orientation Towards Experience, (Art) Product and Mediation. Concrete Approaches to Project-Based Teaching in the Field of Media Pedagogy and Cultural Education in Empirical Comparison

\begin{abstract}
The central position of the project method is emphasized both within action-oriented media pedagogy and in the field of cultural education. However, neither the purposes of using this approach nor it's unique features are clearly explicated. With the intention of supporting media and cultural mediators in the conception of their interventions, this
\end{abstract}


article examines the research question of the potential of concrete approaches to projectbased teaching with regard to specific achievable objectives in the discussed area. In order to answer that question, this contribution presents and analyzes 10 scientifically accompanied workshops, which extended over several weeks and often months and which were mostly carried out by media artists at an urban secondary school. In the course of this, three approaches to working at the intersections between media pedagogy and cultural education are extracted and described in detail: the orientation towards experience, the focus on the (art) product and the emphasis on mediation aspects. Finally, these approaches are compared with respect to the research question, which results in the pointing out of some practical consequences.

\section{Problemstellung, Zielsetzung und Forschungsfrage}

In jenem Bereich der Medienpädagogik, in dem die aktive bzw. kreative Medienarbeit eine zentrale Rolle spielt, werden projektorientierte Vermittlungsformen als der «Königsweg» betrachtet (vgl. Zeidler 2009, 245). Dieter Baacke, der als der «Vater» dieser Strömung gilt (Ruge 2017, 116), beharrte sogar darauf, dass Medienkompetenz «nur und ausschließlich über Projektarbeit umzusetzen» sei (Baacke 1999, 35). Eine ähnliche Fokussierung ist ebenso auf dem Gebiet der Kulturellen Bildung zu verzeichnen, in dem sämtliche Praxisinterventionen zumeist unter dem Begriff der Projektarbeit subsumiert werden (siehe z. B. Bundeszentrale für politische Bildung o. J.). Der Einsatz derartiger Verfahren wird nicht nur in ausserschulischen kulturpädagogischen Tätigkeitsfeldern präferiert. Von der Einladung von Künstlerinnen und Künstlern zur Durchführung von Projekten verspricht man sich auch, dass «frischer Wind (...) in die Schulen geweht» wird, womit «wichtige Impulse für die Schulentwicklung» einhergehen sollen (Schega 2005, 10).

Dem enormen Stellenwert des Projektzugangs zum Trotz scheint innerhalb beider Disziplinen wenig Klarheit darüber zu herrschen, welche konkreten medien- und/ oder kulturpädagogischen Ziele mit seiner Hilfe verwirklicht werden könnten und worin die Spezifik einer solchen Methode (im Vergleich zu anderen Vermittlungsverfahren) bestehen soll. So werden in medienpädagogischen Nachschlagewerken als die zentralen Intentionen der Projektarbeit die Förderung individueller und gesellschaftlicher Handlungs- und Gestaltungsmöglichkeiten (Schell 2008, 590) sowie die Steigerung der Fähigkeit der Mitwirkenden zur Selbstorganisation und zum problemlösenden Lernen (Ziedler 2009, 245) angegeben. Im Feld der Kulturellen Bildung wird der Ansatz noch weiter gefasst - «als Gegenbewegung zur formalen Wissensvermittlung», die auf das "ganzheitliche und sinnbetonte Erfahren» ausgerichtet ist (Lowinski 2007, 161). Ebenso sind Positionierungen zur Frage nach den besonderen Merkmalen der Projektmethode höchst allgemein gehalten. In medienpädagogischen Publikationen werden dazu Schlagworte wie handelndes und exemplarisches 
Lernen sowie Zielgruppen-, Wirklichkeits- und Produktorientierung angeführt (vgl. Schell 2008, 591; Ziedler 2009, 245). Kulturpädagogischen Veröffentlichungen ist zu entnehmen, dass sich Projekte "für zahlreiche Themen und Bildungsprozesse» (Lowinski 2007, 161) eignen und sich «durch interdisziplinäres und interkulturelles Arbeiten sowie durch wechselnde Arbeitsformen von der Einzelarbeit über die Arbeit mit PartnerInnen und in Kleingruppen bis zum Zusammenwirken in der Gesamtgruppe» (Ellermann und Flügge-Wollenberg 2013) auszeichnen. Dass solche Informationen Menschen, die selbst derartige Interventionen durchzuführen planen, kaum Orientierungshilfen bieten, ist offensichtlich.

Genau darin, Medienpädagoginnen und -pädagogen, Künstlerinnen und Künstler, Kulturvermittlerinnen und -vermittler sowie Lehrende bei der Konzeption ihrer Praxisprojekte zu unterstützen, besteht die Intention dieses Artikels. Deswegen wird hier folgender Forschungsfrage nachgegangen: Welche spezifischen Ziele der Arbeit an den Schnittstellen zwischen der Medienpädagogik und der Kulturellen Bildung lassen sich unter Einsatz welcher konkreten Zugänge zum Projektunterricht am ehesten erreichen?

\section{Projektanlagen und Forschungsdesigns}

Im vorliegenden Beitrag erfolgt die Darstellung von insgesamt zehn medien-kulturpädagogischen Projekten, die in drei Reihen bzw. Teilstudien strukturiert sind. Bevor die Spezifika (und damit die Unterschiede) der jeweiligen Anlagen sowie die einzelnen Untersuchungsdesigns behandelt werden, gilt es, auf die zwei wichtigsten Gemeinsamkeiten hinzuweisen.

Die erste besteht darin, dass der überwiegende Teil der besprochenen Interventionen in einem wöchentlich (doppelstündig) abgehaltenen Unterricht an einer städtischen Haupt- bzw. Mittelschule stattfand - im Wahlpflichtfach «Kreative Mediengestaltung, das der Autor konzipierte und seit vielen Jahren in der 7. und 8. Schulstufe lehrt (Altersgruppe 12-15, Gruppengrösse 10-15). Der zweite übergreifende Aspekt ergibt sich daraus, dass hinsichtlich Forschungsverfahren die Rahmenmethodologie Design-Based Research (DBR) eine zentrale Rolle spielte. Bei der ersten und in der ersten Hälfte der zweiten Projektreihe kam sie in ihrer «klassischen` Art zum Einsatz: erstens in Form der Arbeit in möglichst authentischen Settings, zweitens als Bemühung um die Entwicklung neuer Praxiszugänge für das jeweilige Vorhaben und drittens im Sinne der Weiterentwicklung solcher Ansätze im Zuge von - auf Zwischenreflexionen basierenden - Iterationen (zur Bedeutung dieser Faktoren für DBR siehe DBR-Collective 2003; Reinmann 2005; Euler und Sloane 2014). Dabei nahm der Autor als Forscher zwar eine beratende und folglich mitgestaltende, aber dennoch vorrangig (fremd-)beobachtende Rolle ein. Bereits gegen Ende der zweiten Hälfte der zweiten Teilstudie kam ein zusätzlicher Zugang hinzu, auf den hin sich im Verlauf der 
dritten Teilstudie der Schwerpunkt verlagerte: die - in neueren Publikationen zu DBR (z. B. bei Reinmann 2019, 138, 142) als besonders vielversprechend hervorgehobene - autoethnografische Herangehensweise, bei der der Verfasser sowie ein weiterer Mitwirkender den Fokus auf die eigene Vermittlungstätigkeit lenkten und in erster Linie mit Selbstbeobachtungen operierten.

Eine weitere Klammer bildet die Verortung des Autors im Pragmatismus und hier in erster Linie sein Aufgreifen der Positionen von John Dewey. Dahingehende Aspekte wirkten sich in der Feldphase des Gesamtvorhabens höchstens indirekt über die Haltungen aus, mit denen an die Beratungs- und Reflexionsgespräche mit den Künstlerinnen und Künstlern herangegangen wurde. Im Verlauf der Analysen und Auswertungen der Materialien rückte diese Erkenntnis- und v. a. die damit zusammenhängende Wissenschaftstheorie jedoch zunehmend ins Zentrum - bis hin dazu, dass der eingesetzte Forschungszugang insgesamt retrospektiv mit dem Begriff der «pragmatistischen Praxisforschung) gefasst wurde (Kurzdarstellung siehe entsprechend benannter Abschnitt in Pasuchin 2022; ausführlich siehe Pasuchin 2021a, 315ff.).

\subsection{Projektanlagen und Erhebungsverfahren}

Die Erhebungsphase der ersten hier untersuchten Projektreihe fand im Rahmen eines auf drei Jahre (2015-2017) anberaumten explorativ angelegten Forschungsvorhabens statt, das der Autor in Kooperation mit einem lokalen Netzwerk von Medienkünstlerinnen und -künstlern durchführte. In dieses Vorhaben waren insgesamt fünf verschiedene Gruppen von Schülerinnen und Schülern sowie sieben Kunstschaffende eingebunden. Die einzigen Vorgaben, die Letztere erhielten, bestanden darin, dass ihr jeweiliger Workshop sich über mehrere Wochen bzw. Doppelstunden zu erstrecken hatte und dabei technische Werkzeuge zum Einsatz kommen mussten. Die Art und Intensität dieses Einsatzes genauso wie die ästhetische Orientierung der Projekte und ihre unterrichtsmethodische Gestaltung standen ihren Leiterinnen und Leitern völlig frei. Die Offenheit der Gesamtanlage zielte - in Übereinstimmung mit dem pragmatistischen Zugang - darauf ab, Überraschendes zu entdecken und entsprechende Aspekte als Ausgangspunkte für vertiefende Analysen zu nutzen. Damit hing auch die Anwendung vielfältiger Erhebungsinstrumente zusammen. Der Schwerpunkt lag auf Videografien (möglichst) aller Prozesse, die im Verlauf des Unterrichts zu verzeichnen waren. Zusätzlich wurden schriftliche Befragungen, Gruppendiskussionen sowie Leitfadeninterviews durchgeführt. Bei den ersten zwei Workshops der hier zunächst behandelten Reihe traten besonders erstaunliche Facetten zutage (auf die in den Schlussfolgerungen eingegangen wird), was den Grund dafür bildete, warum der Künstler Stefan Luft (alle Namen wurden anonymisiert) um eine dritte Abhaltung gebeten wurde. Sein erstes (Kurz-)Projekt erstreckte sich von Anfang bis Ende Juni 2015, sein zweites von September bis November 2015 und das dritte von Januar bis März 2017 (7, 15 und 18 Unterrichtseinheiten). 
Die ersten beiden Workshops der zweiten Teilstudie, um die es im vorliegenden Beitrag geht, fanden ebenso im Rahmen des gerade besprochenen Forschungsvorhabens statt (erster von November 2015 bis Februar 2016, 19 Schulstunden, und zweiter von Mai bis Juni 2017, 18 Unterrichtseinheiten). Sie wurden mit der zweiten und dritten Gruppe durchgeführt, mit der der erwähnte Künstler arbeitete, wobei die gleichen Erhebungsinstrumente zum Einsatz kamen. Anschliessend wurde der Zugang des Paares, das diese Workshops gestaltete (Maria Rešić und Fritz Stöllinger) zu einem neuen Forschungsvorhaben weiterentwickelt, an das die Leiterin und der Leiter aus einer (eher praktischen) künstlerischen bzw. künstlerisch-forschenden Perspektive herangingen, während der Autor dieses Beitrags (vorrangig wissenschaftliche) medien- und kulturpädagogische Aspekte im Blick hatte. Beim ersten Unterprojekt, das das gesamte Schuljahr 2018/19 einnahm (60 Unterrichtseinheiten zzgl. Exkursion, Aufführung und Abschlussreflexion), nutzte er als Erhebungsinstrument vorrangig die nicht-teilnehmende Beobachtung. Dabei protokollierte der Verfasser die Interaktionen zwischen den mitwirkenden Jugendlichen sowie der Künstlerin und dem Künstler und ebenso seine eigenen Vor-, Zwischen- und Nachbesprechungen mit Rešić und Stöllinger. Zusätzlich wurden von einer externen Expertin zwei Gruppendiskussionen mit ausgewählten Schülerinnen und Schülern durchgeführt. Für das zweite Unterprojekt, das im Schuljahr 2019/20 vom Mitte September bis Anfang März abgehalten wurde (46 Schulstunden zzgl. weiterer Dreharbeiten), wechselte der Autor in die Rolle des teilnehmenden (Selbst-)Beobachters. Ausgehend von einem gemeinsam mit Rešić und Stöllinger entwickelten Rahmenkonzept leitete er den Unterricht mit zwei Teilgruppen und dokumentierte die Prozesse in der Art eines Forschungstagebuches. ${ }^{1}$

Damit ging bereits eine Schwerpunktverlagerung in Richtung Autoethnografie einher - ein Ansatz, der gezwungenermassen die dritte Projektreihe prägte, da ihre Erhebungsphase in die Zeit der (ersten) Corona-Beschränkungen fiel, in der zuerst kein Präsenzunterricht möglich war und danach keine sschulfremden> Personen daran beteiligt werden konnten. Zunächst erprobte der Autor ein Vermittlungskonzept im Rahmen eines Lehrpraxis-Kurses, der in Distanzlehre mit (Musik-)Studierenden stattfand. Für diese Lehrveranstaltung erstellte und in ihrem Zuge evaluierte sowie weiterentwickelte Materialien wurden am Schluss des Schuljahres 2019/20 im Präsenzunterricht an der selben Schule eingesetzt, an der die vorhin beschriebenen Workshops stattfanden (insgesamt sieben - auf zwei Untergruppen aufgeteilte Doppelstunden). Sowohl zu den Vorgängen im Zusammenhang mit der Gestaltung der Unterlagen als auch zu den Beobachtungen, die der Verfasser im Verlauf des Unterrichts an der Universität und der Schule machte, führte er Forschungstagebücher,

1 Die Arbeit der Kunstschaffenden wurde in dieser Phase von einem externen Mitarbeiter beobachtet. Da sich ab diesem Zeitpunkt der forschungsmethodische Fokus auf Autoethnografie verlagerte, werden die von ihm erstellten Protokolle hier nicht berücksichtigt. 
in die ebenso Zusammenfassungen der Reflexionsgespräche mit den Mitwirkenden integriert wurden sowie Analysen ihrer (Zwischen-)Produkte einflossen. Dabei legte er bewusst einen Fokus auf die eigenen subjektiven Eindrücke. Um eine zusätzliche Perspektive einzubeziehen, erfolgte die Einbindung eines weiteren Lehrenden in das Vorhaben. Letzterer nutzte in der Zeit des zweiten Lockdowns im Winter und Frühjahr 2021 die Materialien, um an einem ländlichen Gymnasium in der 9. Schulstufe ein Projekt mit seinen vier (ausschliesslich männlichen) Schülern im Fach Gruppenmusizieren in Distanzlehre abzuhalten, ${ }^{2}$ worüber auch er ein Forschungstagebuch führte. Abschliessend wurde er vom Autor interviewt.

\subsection{Aufbereitungsansätze und Auswertungszugänge}

Aufgrund der explorativen Anlage des Forschungsvorhabens, das den Ausgangspunkt der vorliegenden Untersuchung bildete, war die Aufbereitung der Daten im Hinblick auf die erste Projektreihe und die erste Hälfte der zweiten Teilstudie höchst aufwendig. Sie bestand aus ‘dichten Beschreibungen〉, bei deren Erstellung zunächst Hinweisen aus dem Umfeld von DBR gefolgt wurde, wonach sich die Praxisforschung als eine «narrative» Forschungskonzeption versteht», die darauf ausgerichtet ist, «eine «Geschichte» zu erzählen und zu begründen» (Moser 2018, 470). Die Abhandlung umfasste nicht nur die Darstellung aller verzeichneten Vorgänge (u. a. Transkripte der Videografien), sondern ebenso die Beleuchtung der Hintergründe sowie Rahmenbedingungen der Arbeit. Die sehr umfangreichen Verschriftlichungen wurden unter Berücksichtigung vielfältiger Blickwinkel kodiert und ausgehend davon - im Zuge der Verfassung einer Habilitationsschrift - in mehreren Durchläufen auf einen ‘publizierbaren` Umfang komprimiert (siehe Pasuchin 2021b, 11-128). Im Vorfeld der Erstellung des vorliegenden Artikels wurden aus diesen Zusammenfassungen jene Passagen extrahiert, die für die Forschungsfrage nach den Potenzialen konkreter Zugänge zum Projektunterricht hinsichtlich damit erreichbarer spezifischer Zielsetzungen relevant erschienen. Betreffend die zweite Hälfte der zweiten Projektreihe und die dritte Teilstudie war der Aufwand für die Aufbereitung bedeutend geringer. Denn einerseits lagen bereits alle Materialien schriftlich vor und andererseits war diesmal von Anfang an klar, dass der Fokus einzig auf der genannten Frage lag. Deswegen wurden in den zur Verfügung stehenden Unterlagen (Beobachtungsprotokolle, Transkripte der Interviews und Gruppendiskussionen sowie Forschungstagebücher) lediglich jene Aussagen mit unterschiedlichen Farben hervorgehoben, die auf diesbezüglich interessante Aspekte hindeuteten. Ihre Zusammenfassung bildete die Grundlage der hier veröffentlichten Beschreibungen und Analysen.

2 Aus den Angaben des Kollegen kann nicht abgeleitet werden, wie viele Unterrichtsstunden das Projekt genau umfasste, da es - aus am Schluss des Punktes 3.3 angegebenen Gründen - einige Verzögerungen und Unterbrechungen gegeben hatte. 
Die Frage danach, welche Auswertungsmethoden zum Einsatz kommen sollen, bleibt innerhalb von Design-Based Research völlig offen. Klar ist lediglich eine grosse Skepsis gegenüber sämtlichen standardisierten sowohl quantitativen als auch qualitativen Verfahren (siehe z. B. Jahn 2014, 3f.). Im Verlauf der Arbeit an der Habilitationsschrift fiel - nach einer langen Suche nach passenden Analyseansätzen und ihrer Erprobung - die Wahl auf zwei ‘basale` Herangehensweisen, deren Grundformen bereits innerhalb der (massgeblich von Dewey beeinflussten) (Ersten Chicagoer Schule) intensiv genutzt wurden: Der erste Zugang besteht in kontrastierenden Gegenüberstellungen bzw. komparativen Analysen von Einzelfällen, die darauf abzielen, «hinter der Gleichartigkeit oder Andersartigkeit der untersuchten Vorkommnisse (...) ein Konzept zu fassen, das das Wesentliche des untersuchten Phänomens (...) herausstellt» (Strübing 2008, 283). Der zweite Ansatz beruht auf der Triangulation unterschiedlicher Perspektiven und Sichtweisen, die Flick $(2010,162)$ zufolge «besonders aufschlussreich [ist], wenn sie zu divergenten Ergebnissen (...) führt, die nach neuen Erklärungen (für die Divergenz) verlangen» (Ausführlich zur Auswertungsmethodik siehe Pasuchin 2021a, 359ff.). Diese Methoden wurden ebenso für die Auswertung der Materialien für den vorliegenden Artikel herangezogen.

\section{Projektansätze und darauf bezogene Beobachtungen}

In diesem Abschnitt erfolgt die Präsentation der Extrakte der Aufbereitungen der erhobenen Materialien zu den drei behandelten Projektreihen. Dabei sind alle Unterabschnitte nach dem gleichen Schema strukturiert: In einem ersten Schritt wird die Zuordnung des Ansatzes zu den einzelnen Arten des Projektunterrichts argumentiert. In einem zweiten findet die Darstellung des jeweiligen Konzepts im Allgemeinen statt. Die dritte Passage ist spezifischen vermittlungsmethodischen Zugängen der involvierten Projektleiterinnen und -leiter gewidmet. Die vierte dient der Zusammenfassung der Beobachtungen der Prozesse mit einem Schwerpunkt auf die Reaktionen und das Agieren der beteiligten Jugendlichen. Abschliessend werden die entstandenen Produkte beschrieben, wobei der Fokus auf ästhetischen Aspekten und darauf bezogenen Resonanzen liegt.

Diese Strukturierung impliziert bereits erste Auswertungsvorgänge. Denn sie zielt darauf ab, die drei (enorm heterogenen) Herangehensweisen an den Projektunterricht miteinander vergleichbar zu machen, weswegen im Verlauf der Erstellung des vorliegenden Textes ständige kontrastierende Gegenüberstellungen vorzunehmen waren. 


\subsection{Erfahrungsorientierung}

Der Grund, warum der Zugang der ersten Projektreihe hier als Erfahrungsorientierung bezeichnet wird, besteht darin, dass der Leiter der entsprechenden Workshops diesen Schwerpunkt selbst immer wieder betonte. Bereits beim Interview, das Stefan Luft im Vorfeld seines ersten Workshops gab, hob er den «Fokus auf Erfahrungen» der Beteiligten als seine «Unterrichtsphilosophie» hervor. ${ }^{3}$ Er bekräftigte, wie wichtig es ihm sei, dass die Mitwirkenden im <learning by doing)-Verfahren Erfahrungen im persönlichen Gestalten erlangten und sich dabei «selber ihre Finger schmutzig machen». Ausserdem wies der Künstler darauf hin, dass für ihn der Prozess und nicht das Produkt im Vordergrund stehe. Das Ergebnis eines Projektes könne inm zufolge im besten Fall dazu dienen, Erfahrungen auszulösen, wobei sich der Wert eines Kunstwerks für ihn grundsätzlich vorrangig an der «Erfahrung, die es liefert» bemesse.

Seine Workshopreihe betitelte Luft «Unsere Welt der Geräusche». Bei den Projekten ging es um die Durchführung von Klangexperimenten mit diversen (Alltags-)Materialien, die mit unterschiedlichen Mikrofonen verstärkt wurden und mit denen die Jugendlichen in der Art eines «Geräuschorchesters» miteinander musizierten. Daraufhin liess der Künstler die Schülerinnen und Schüler mithilfe der Kombination der Aufzeichnungen solcher Prozesse «Minutenstücke» am Computer gestalten. Als Vorund Zusatzübungen forderte er die Beteiligten unter anderem auf, im Unterrichtsraum still sitzend auf die Klangumgebung zu lauschen und das Gehörte grafisch zu notieren, sich gegenseitig nur unter Einsatz ihrer Hände Klangverläufe an der Grenze des Hörbaren vorzuführen oder Umweltsounds im Umfeld der Schule aufzunehmen, auf deren Basis sie «Geräusche-Landkarten» von ihrem Viertel zeichnen sollten.

Auf der inhaltlichen Ebene und bezüglich der Umsetzungsaspekte hatten die Mitwirkenden kaum Mitgestaltungsoptionen, was auch daran zu erkennen war, dass die drei Workshops von Luft immer nach einem ähnlichen Schema abliefen. Hinsichtlich Unterrichtsmethoden war bei der Arbeit des Künstlers jedoch eine sehr grosse Vielfalt und ihr häufiger Wechsel zu beobachten. Auf Fragerunden (z. B. nach dem Unterschied zwischen Tönen und Geräuschen oder dazu, ob es «absolute Stille» gebe) schlossen Diskussionen an, denen dazu passende Präsentationen audiovisueller Beispiele folgten. Daraus resultierten (zumeist kurze) theoretische Inputs, die in Übungen und Aufgaben mündeten, deren Ergebnisse sofort gemeinsam besprochen wurden, was zu Erläuterungen zu den gerade erlebten Phänomenen führte, woraufhin der Kreislauf oft von vorne begann. Ein weiteres spezifisches Merkmal des Vermittlungsansatzes des Künstlers bestand darin, dass er die Jugendlichen vieles ausprobieren liess, ohne ihnen (Qualitäts-)Kriterien vorzugeben, an denen sie sich orientieren sollten. Z. B. wies er sie zu Beginn der Phasen des «Geräuschorchesters» jedes Mal an, völlig frei mit den Materialien zu experimentieren, was oft in höchst

3 Ohne Quellenverweise in Anführungszeichen angegebene Aussagen im vorliegenden Abschnitt und den folgenden Passagen entstammen den Transkripten von Interviews oder Videografien. 
chaotischen Klangzuständen mündete. Erst bei weiteren Durchläufen dieser Übung im Zuge seines zweiten und v. a. des dritten Workshops agierte Luft als eine Art Dirigent, woraus eine stärkere Strukturierung der akustischen Prozesse resultierte. Besonders deutlich wurde seine 〈lockere» Herangehensweise in den Etappen der grafischen Notation des Wahrgenommenen und in jenen, in denen aus den Aufnahmen des "Geräuschorchesters» am Computer Stücke zu arrangieren waren. Denn in beiden Fällen erklärte er nur kurz, worauf bei der Ausführung der Aufgaben zu achten sei. Auf die daraufhin häufig seitens der Mitwirkenden gestellte Frage, was sie konkret tun sollen, antwortete der Künstler, dass es bei so einer Tätigkeit «kein Richtig und kein Falsch» gebe. Zu den (Zwischen-)Ergebnissen der Beteiligten äusserte er sich ihnen gegenüber fast immer sehr lobend.

Die Reaktionen der Schülerinnen und Schüler und ihr Agieren im Verlauf der Projekte wichen von Gruppe zu Gruppe sehr stark voneinander ab. Beim ersten Durchgang stiess Lufts Ansatz grösstenteils auf Ablehnung, die bei manchen in absoluter Verweigerung mündete. Hingegen schienen die meisten Jugendlichen, die die erste Wiederholung des Workshops erlebten, davon fasziniert zu sein. Das liess sich an ihrer hochengagierten und freudvollen Mitwirkung beobachten und zeigte sich auch an ihren überwiegend sehr positiven (und z. T. überschwänglichen) Rückmeldungen im Rahmen von Fragebögen sowie Interviews. Dabei gaben fast alle nicht nur an, von diesem Projekt und seinem Leiter begeistert zu sein, sondern beim Workshop auch viel Neues und Spannendes erfahren bzw. gelernt zu haben. In der dritten Gruppe herrschte zu Beginn des Projektes eine ähnlich enthusiastische Stimmung vor wie in der zweiten. Jedoch legten die Teilnehmenden daraufhin zunehmend kindliches Benehmen an den Tag. Der Versuch des Künstlers, der Bitte des Autors nachzukommen und am Schluss seines letzten Workshops eine (auf Video aufgezeichnete, öffentlich präsentierbare) Aufführung des "Geräuschorchesters» vorzunehmen, ${ }^{4}$ scheiterte an Disziplinproblemen. Abgesehen von der allgemeinen Gruppendynamik könnte das daran gelegen sein, dass Luft in dieser Phase von seinem Grundkonzept sowohl im Hinblick auf die Ästhetik (siehe unten) als auch die Unterrichtsmethodik abwich. Letzteres, weil er sich - offensichtlich aufgrund der Anforderung, etwas «Vorzeigbares) zu generieren - viel kritischer über die Beiträge der Beteiligten äusserte.

Auf jeden Fall ist es bei der ersten Projektreihe kaum möglich, von einem (End-) Ergebnis bzw. Produkt zu sprechen. Am ehesten können noch die «Minutenstücke» entsprechend bezeichnet werden, die die Jugendlichen am Computer gestalteten und innerhalb der Gruppe präsentierten. Beim ersten und v. a. dem zweiten Workshop klangen sie nach zeitgenössischer elektroakustischer Musik - ein Umstand, mit dem der Künstler in Interviews begründete, warum er sie als qualitativ besonders hochwertig empfand. Am Schluss der zweiten Durchführung und noch mehr bei der

4 Hinter dieser Bitte stand die Kritik seitens der Institution, die die Workshops finanzierte, dass in ihrem Verlauf zu wenig ‘herauskommen` würde. 
dritten operierte Luft stärker mit Beats, die an populäre Musikproduktionen erinnerten. Dabei war seinen Rückmeldungen gegenüber dem Autor zu entnehmen, dass er die Ergebnisse hinsichtlich der Ästhetik eher geringschätzte. Überraschenderweise waren die Reaktionen der Mitwirkenden ähnlich: Während sie die avantgardistisch anmutenden Kreationen der Kolleginnen und Kollegen grösstenteils mit Begeisterung aufnahmen, schienen die stärker an die Jugendkultur angelehnten Stücke sie kalt zu lassen.

\subsection{Ausrichtung am (Kunst-)Werk}

Die zentrale Rolle, die das (Kunst-)Werk und damit das Produkt im Gesamtkonzept der zweiten Projektreihe spielte, ist zahlreichen Aussagen von Maria Rešić und Fritz Stöllinger zu entnehmen, die sie im Verlauf mit ihnen geführter Interviews machten. Unter anderem betonten sie, es gehe ihnen hauptsächlich darum, mit den Jugendlichen «etwas Besonderes (...) ein interessantes Kunstwerk» zu gestalten. Prozessorientierte Ansätze setzten sie mit der Taktik «das, was herauskommt, kommt heraus» gleich, wogegen für sie die Qualität des Ergebnisses essenziell sei. Auf die Frage danach, wie sie vorhätten, eventuellen Motivationsproblemen seitens der Schülerinnen und Schüler zu begegnen, verwiesen die Kunstschaffenden auf ihre Strategie, sie immer wieder daran zu erinnern, «dass es darum geht, am Ende ein Kunstwerk zu haben und das gemeinsam zu erreichen».

Die Workshops von Rešić und Stöllinger zielten auf die Entwicklung von Mixed Reality-Games $a b-d$. h. von Spielen, in denen sich unmittelbare Erfahrungen mit medienvermittelten Erlebnissen vermischen. Die Spielenden sollten in einem realens Setting (z. B. im Schulgebäude oder seiner Umgebung) immer wieder an Punkte kommen, an denen sie in einem (bei den ersten drei Produktionen am Handy-Display, bei der letzten auf einer Leinwand eingespielten) Video die Erklärung der jeweiligen Situation sowie weitere Anweisungen erhielten. Die Ergebnisse wurden im Verlauf der Projektreihe auf der Umsetzungsebene jedes Mal komplexer. Während beim ersten Durchgang eine lineare ‘Schnitzeljagd entstand, wurden den Spielenden in Folge immer mehr verschiedene Optionen angeboten, wobei im letzten Projekt auch «live` zu bewältigende Herausforderungen (wie z. B. Geschicklichkeitsspiele) hinzukamen. Die Themen, um die es ging, reichten vom Aufdecken geheimer Atomexperimente im Stadtviertel über die Abwehr eines Zombie-Angriffs auf die Schule bis hin zur Bemühung, als Influencerin bzw. Influencer besonders erfolgreich zu sein. Die Aufgaben der beteiligten Schülerinnen und Schüler bestanden darin, diese Themen vorzuschlagen, die Grundideen zu einer Geschichte und danach zu einzelnen Szenen weiterzuentwickeln, die konkreten Spielregeln auszuarbeiten und v. a. die audiovisuellen Einspielungen zu gestalten. Dazu machten sie Bild- und Tonaufnahmen (bzw. luden sich entsprechende Materialien aus dem Internet herunter) und bearbeiteten sie am Computer. 
In den Anfangsphasen unterrichteten Rešić und Stöllinger jeweils die gesamte Gruppe, indem sie allgemeine Einführungen (u. a. zu künstlerischen Games und zu Spielregeln) vornahmen, wozu sie überwiegend Vortragsformate inklusive Beispielpräsentationen nutzten. Ausserdem liessen sie die Mitwirkenden Spiele ausprobieren und Ideen sammeln. Sobald es um die Umsetzung darauf aufbauender (von Rešić und Stöllinger selbst im Detail ausformulierter) Konzepte ging, fand ein Wechsel zur Arbeit in immer kleineren Gruppen statt - bis dahin, dass die Jugendlichen im zweiten und erst recht im dritten Projekt dieser Reihe zum Schluss vorrangig Einzelaufträge zu bewältigen hatten. Die daraus resultierende enorme Individualisierung erleichterte den Kunstschaffenden einerseits, auf die persönlichen Interessen und die (stark divergierenden) Arbeitstempi der Teilnehmenden einzugehen. Andererseits erschwerte ihnen das nach Beobachtung des Autors jedoch, jeder und jedem zu erklären, was, wozu und v. a. wie es zu tun war. Das könnte wiederum den Grund dafür bilden, warum zahlreiche zentrale Elemente, die in die Endprodukte einflossen, von Rešić und Stöllinger selbst sowie (v. a. bei ihrem dritten Projekt) von ihren Mitarbeiterinnen und Mitarbeitern fertiggestellt wurden. In der (im Endeffekt gescheiterten) Hoffnung, die Schülerinnen und Schüler intensiver in die konkrete Ausgestaltung der Projektergebnisse einzubeziehen, schlug der Autor vor, sie im vierten Durchlauf von Anfang an drei Untergruppen bilden zu lassen. Diese sollten auf Basis eines gemeinsamen Rahmenkonzepts unterschiedliche Spiele entwickeln, während sie abwechselnd von jeweils einer Lehrperson betreut werden sollten, wobei sich der Verfasser selbst (neben Rešić und Stöllinger) als eine davon anbot.

Auch innerhalb der zweiten Projektreihe wichen die Reaktionen der Teilnehmenden und ihr Agieren im Verlauf der Workshops stark voneinander ab, wobei die Unterschiede nicht so extrem waren wie im Zuge der Massnahmen von Luft. Bei der ersten Gruppe, mit der Rešić und Stöllinger arbeiteten (die die zweite Gruppe war, mit der Luft seine Workshops durchführte), war zu Beginn vorrangig Desinteresse zu beobachten. Nach Interpretation des Autors hing dies in erster Linie mit einer stark auf Vorträge fokussierten Unterrichtsweise der beiden Kunstschaffenden zusammen. Sobald die Schülerinnen und Schüler aktiver mitwirken konnten, machten sie das mit grosser Freude, die sich zum Schluss des Projektes bis hin zur Begeisterung steigerte. Der zweite Workshop von Rešić und Stöllinger verlief bezüglich der (Re-) Aktionen der Jugendlichen genau «spiegelverkehrt). Nach der Anfangsphase, in der fast alle Beteiligten einen hochmotivierten Eindruck machten, legten einige von ihnen mit der Zeit zunehmend ähnlich destruktive Verhaltensweisen an den Tag, wie sie bei der gleichen Gruppe beim dritten Workshop von Luft zu verzeichnen waren. Im Rahmen des dritten Projektes von Rešić und Stöllinger war das «Motivationstief) bei den meisten in der Mitte der Laufzeit zu beobachten, was daran gelegen haben könnte, dass in dieser Etappe nur jene (wenigen) Teilnehmenden, die besonders engagiert mitwirkten, intensiv in die zentralen Prozesse einbezogen wurden, während 
der überwiegende Rest eher unwichtige und mechanische Aufgaben zu erledigen hatte. Bei der ersten Untergruppe, mit der der Autor im vierten Durchlauf an der Ideensammlung für das Projekt und der Entwicklung des Konzeptes arbeitete, brachte eine Schülerin zahlreiche Vorschläge ein und erstellte auch ausserhalb des Unterrichts eigene Skizzen. Ihre drei (männlichen) Kollegen machten jedoch grösstenteils unwillig mit und trugen insofern wenig zum Projektfortschritt bei. Die zweite vom Verfasser angeleitete Untergruppe bestand aus drei Mädchen, von denen eine das Lernen in allen Fächern verweigerte und im weiteren Verlauf die Schule abbrach. Der Versuch, daraus resultierenden Problemen zu begegnen, bestand darin, einer ihrer Kolleginnen, die an der Tätigkeit (v. a. im Hinblick auf Bildgestaltung) interessiert war, zusätzliche Einzelbetreuungen anzubieten, in deren Rahmen einige Materialien fertiggestellt werden konnten. Beim Unterricht mit allen drei Teilnehmerinnen fanden vorrangig Ideensammlungen und die Arbeit an Entwürfen statt, von denen ausgehend der Verfasser - aus dem Wunsch heraus, das Werk zu 〈retten〉 - selbst das Endprodukt finalisierte.

Die Präsentationen der Ergebnisse waren jedes Mal aufwendiger gestaltet. Das erste Spiel probierten lediglich jene Schülerinnen und Schüler aus, die es entwickelten, das zweite spielte fast die gesamte Schule im Rahmen eines Projekttages. Die Aufführung des dritten Spiels wurde (nach einem Testdurchlauf an der Schule) genauso wie jene des vierten an einer renommierten Stätte für Kulturveranstaltungen vorgenommen, wobei die Schülerinnen und Schüler (u. a. als Spielleiterinnen und -leiter) intensiv in die beiden Präsentationen eingebunden waren. Hinsichtlich ästhetischer Aspekte ging mit dem Anspruch, ein grösseres Publikum zu erreichen, eine offensichtliche ‘Professionalisierung einher - während die Ergebnisse zu Beginn nach spontanem ¿do it yourself) aussahen, wirkten sie im Vergleich dazu zum Schluss hin zunehmend wie durchgestylte «Hochglanzproduktionen`. Abgesehen von der bereits erwähnten Intensivierung der Unterstützung durch das Projektteam und externe Fachleute hing dies auch mit der Übernahme und dem Remix zahlreicher Elemente zusammen, die aus der populären Jugendkultur übernommen waren (z. B. Comicfilter, Memes und auf YouTube gefundenes Footage). Die zwei letzten Veranstaltungen stiessen auf grosse Aufmerksamkeit der lokalen Presse. Mehrere Zeitungs- und Rundfunkreporterinnen und -reporter kamen in den Unterricht, um die Jugendlichen zu interviewen. Diese Wertschätzung, der Veranstaltungsort und das Interesse eines <externen) Publikums an ihrer Arbeit wirkten auf die Beteiligten sichtlich motivierend. Bei den Aufführungen waren ihnen grösste Konzentration und Stolz anzumerken. Dieser steigerte sich im Nachhinein, als sie erfuhren, dass die Projektergebnisse (Haupt-)Preise bei mehreren Wettbewerben erhalten hatten. 


\subsection{Fokussierung auf Vermittlungsaspekte}

Im Rahmen von Reflexionsgesprächen mit Luft und v. a. mit Rešić und Stöllinger bemängelte der Autor immer wieder, dass die Kunstschaffenden den mitwirkenden Jugendlichen zu wenig erklären würden, wie (und wozu) die ihnen gestellten Aufgaben zu bewerkstelligen seien. Im Verlauf der Selbstbeobachtungen bei der Durchführung eigener Projekte kristallisierte sich heraus, dass ihr Schwerpunkt gerade auf solchen Erläuterungen lag - so sehr, dass der Ansatz als Vermittlungsorientierung bezeichnet werden kann. Dazu entwickelte der Verfasser im Zuge seiner langjährigen medienkulturpädagogischen Tätigkeit zahlreiche ‘kleinschrittig) aufgebaute Projektarten, die darauf abzielen, dass die Beteiligten jeden Arbeitsvorgang sowie dessen Sinn im Gesamtkontext genau nachvollziehen und davon ausgehend eigenständige Beiträge zum Endprodukt leisten.

Für die dritte Teilstudie wurde davon ein Zugang ausgewählt, der auf Videoaufnahmen basiert, auf denen einzelne Personen zu sehen sind, die Klänge oder Geräusche ausführen. Diese Aufnahmen werden in einem Bearbeitungsprogramm auf immer kürzere Sequenzen zugeschnitten, aus deren Kombination die Gestaltung von Rhythmen erfolgt, während auf der visuellen Ebene (aufgrund der Bewegungen, die die Ausführenden machen) eine Art Tanzvideo entsteht. Im konkreten Fall bestand das Hauptmaterial aus Silben eines (von den Teilnehmenden erfundenen) Satzes, den die Mitwirkenden einzeln mit ihren Laptops aufnahmen und mithilfe des Videoschnitts sowie von Effekten zu musikalischen Verläufen zusammensetzten, die zum Teil nach Rap klangen. Den Corona-Bedingungen entsprechend diente als Rahmenhandlung eine (ironisch verfremdete) Videokonferenz.

Da sämtliche Unterrichtsunterlagen - Lernvideos, Handouts, Musterbeispiele und Übungsdateien - online veröffentlicht sind (siehe Pasuchin 2020), ist es nicht notwendig, das Vermittlungskonzept im Detail vorzustellen. Zu erwähnen sind lediglich zwei Aspekte: Der erste betrifft die spezielle (Corona-)Situation, die der Autor zum Anlass nahm zu versuchen, ein Problem zu beheben, das eine Schülerin und ein Schüler im Zuge einer der Gruppendiskussionen zur zweiten Projektreihe ansprach. Es bestand darin, dass sein Unterricht (im Vergleich zu jenem von Rešić und Stöllinger) für jene, die die Aufträge schnell erfassten und erledigten «voll langweilig» war, weil sie immer warten mussten, bis den «Langsamen» alles erklärt wurde. Die Lernvideos, die für die hier beschriebene konkrete Umsetzung erstellt wurden, dienten dem Ziel (sowohl im Rahmen des Distanz- als auch des Präsenzunterrichts) den Teilnehmenden zu ermöglichen, die Grundaufgaben in einer individuellen〉 Geschwindigkeit durchzuführen und sich - im Falle, dass sie früher fertig wurden - persönlich beraten zu lassen sowie davon ausgehend in Details zu vertiefen. Der zweite Aspekt betrifft das Vermittlungskonzept im Allgemeinen, wobei dem Verfasser erst im Zuge der Selbstreflexionen auffiel, dass es grundsätzlich auf Wechseln zwischen dem Imitieren vorgegebener Schritte und eigenem kreativen Handeln beruht. Auch 
beim Endprodukt dieses Projektes war der - sich mehrmals wiederholende - kurze Refrain (von den Ideen der Teilnehmenden abgeleitet) vom Autor vorgefertigt. Die den Mitwirkenden gestellte Hauptaufgabe bestand darin, jeweils eine Strophe unter vorrangigem Einsatz des von ihnen aufgenommenen Materials selbstständig zu gestalten, wofür sie (zunächst) eher 〈lockere〉 Regeln erhielten.

Im Verlauf der Beobachtung der entsprechenden Prozesse und hier v. a. bei den Analysen der abgegebenen (Zwischen-)Produkte erwies sich gerade Letzteres als grosse Herausforderung - bereits im Hinblick auf die Musikstudierenden, die am Vorprojekt teilnahmen. Denn viele von ihnen wirkten mit der eigenständigen Entwicklung eines Rhythmus, der (von aussen betrachtet) sowohl musikalisch sinnvoll als auch abwechslungsreich genug war, überfordert, weswegen sie bei der Fertigstellung viel (Online-) Unterstützung benötigten. Ausgehend von dieser Erfahrung wurden die Gestaltungsregeln für die Arbeit mit den Schülerinnen und Schülern stark vereinfacht, was auch deswegen notwendig war, weil aufgrund des Corona-bedingten «Wechselunterrichts> bei einer Untergruppe nur drei und bei der anderen vier Präsenz-Doppelstunden für das Projekt zur Verfügung standen (während mindestens sechs notwendig gewesen wären). Die eindeutigen Richtlinien, die Lernvideos und die Hilfe, die der Autor einzelnen Jugendlichen bei ihrer Umsetzung anbieten konnte, schienen ihnen die Tätigkeit zu erleichtern. Bis auf eine Ausnahme schafften es alle, sowohl die ästhetisch-formalen als auch die technischen Anforderungen zu erfüllen, wobei die «Langsameren) gut mitkamen, während den «Schnelleren` keine (vorhin angesprochenen) Motivationsmängel anzumerken waren. Jedoch schränkten die klaren Vorgaben die Kreativität der Schülerinnen und Schüler offensichtlich beträchtlich ein, was daran zu erkennen war, dass ihre Ergebnisse (im Gegensatz zu jenen des Vorprojektes mit den Studierenden) grösstenteils sehr ähnlich klangen. Dem anderen Lehrenden, der die Materialien in seinem Unterricht am ländlichen Gymnasium einsetzte, wurde deswegen geraten, bei den Kriterien für die Gestaltung der Rhythmen (wieder) etwas grössere Interpretationsspielräume zu lassen. Seine Eintragungen im Forschungstagebuch und Äusserungen beim Interview können so ausgelegt werden, dass das zu einigen Irritationen bei den vier beteiligten Schülern führte. Eine alternative bzw. weitere Erklärung für die vielen Schwierigkeiten, die im Verlauf seines Projektes zu verzeichnen waren, könnte darin bestehen, dass es in die Phase des zweiten Lockdowns fiel und deshalb vollständig im Distanzunterricht abgehalten wurde. Zwei der Jugendlichen machten (nach der Überwindung anfänglicher Kommunikationshürden) dabei höchst aktiv mit und brachten als sehr kreativ bewertete Beiträge ein, während ein anderer wenig beitrug und der vierte kaum erreichbar war. Auf die Lust der Teilnehmenden, bei der Arbeit ‘am Ball zu bleiben`, wirkte sich eventuell auch die Tatsache kontraproduktiv aus, dass der Lehrer wegen Erkrankungen das Projekt mehrmals (und einmal für längere Zeit) aussetzen musste. Ausserdem ist seinen Aussagen zu entnehmen, dass er aufgrund geringer Vorkenntnisse im Hinblick 
auf Videoschnitt mit der technischen Umsetzung des Projektes selbst häufig überfordert war und insofern den Schülern bei entsprechenden Problemen nicht immer helfen konnte.

Die Endprodukte der Projekte, die an der Kunstuniversität und an jenem Gymnasium, von dem zuletzt die Rede war, durchgeführt wurden, sind im Internet publiziert (siehe Pasuchin 2020), was den Leserinnen und Lesern ermöglicht, sich selbst ein Bild von ihrer ästhetischen und formalen Gestaltung zu machen. Die Rückmeldungen der Studierenden zum Ergebnis ihres Vorhabens waren durchwegs sehr positiv - genauso wie die Feedbacks ihrer Kommilitoninnen und Kommilitonen sowie mehrerer Dozierender, die es auf YouTube oder Facebook sahen. Im Zuge der Abschlussreflexion wurde seitens der Studierenden mehrmals hervorgehoben, dass sie sich zu Beginn nicht vorstellen konnten, dass das Video «so witzig» werden und «so gut ankommen» würde. Ähnliche Reaktionen waren bei den Teilnehmenden des Projekts am Gymnasium und in dessen Lehrenden-Kollegium zu verzeichnen. Hingegen wirkten die meisten Schülerinnen und Schüler, mit denen der Autor in seinem Unterricht an der Haupt- bzw. Mittelschule arbeitete, von der Ästhetik ihrer Produkte irritiert. Einige bezeichneten die Videos als «krank» und alle sprachen sich gegen eine Publikation im Internet aus. Jedoch ist anzumerken, dass Jugendliche, die die gleiche Schule besuchten, sich bei früheren Durchführungen ähnlicher Projekte (für die bedeutend mehr Zeit zur Verfügung gestanden hatte) mehrmals für eine Veröffentlichung der Ergebnisse entschieden und - v. a. in Folge von Wettbewerbserfolgen - auf ihre Werke sehr stolz zu sein schienen.

\section{Schlussfolgerungen im Hinblick auf die Forschungsfrage}

Dieser Abschnitt ist der Beantwortung der Forschungsfrage danach gewidmet, mit welchen konkreten Zugängen zum Projektunterricht sich welche Ziele bei der Arbeit an den Schnittstellen zwischen Medienpädagogik sowie Kultureller Bildung am ehesten erreichen lassen. Im Vorfeld ist anzumerken, dass ein qualitativer Vergleich von Einzelfällen - so viele davon und wie genau auch immer sie untersucht werden niemals zu generalisierbaren Aussagen führen kann. Unter Einsatz einer derartigen Methode ist es höchstens möglich, «begründete Annahmen` bzw. Hypothesen bzgl. allgemeiner Tendenzen zu generieren und zur Diskussion zu stellen. Die zentrale Herangehensweise, um im Zuge der vorliegenden Analysen zu solchen Schlussfolgerungen zu gelangen, bestand in einer Schwerpunktsetzung auf die Triangulation unterschiedlicher Perspektiven sowie Sichtweisen und hier vorrangig auf jene Aspekte, die im Widerspruch zu den eigenen Vorannahmen des Autors standen und/oder ihn selbst erstaunten. 
Wie bereits erwähnt, waren im Verlauf der ersten zuvor behandelten, als Erfahrungsorientierung bezeichneten Projektreihe die grössten entsprechenden Überraschungen zu erleben. Denn bei ihrem zweiten Durchlauf (und über weite Strecken ebenfalls beim dritten) setzten sich die teilnehmenden Jugendlichen höchst aktiv sowie mit offensichtlicher Faszination mit einer Ästhetik auseinander, die im Kontrast zu allem steht, womit sich Bourdieu $(2014,23)$ zufolge "die (gewöhnlichen Menschen in ihrem (gewöhnlichen) Dasein herumschlagen.» Davon kann abgeleitet werden, dass ein besonderes Potenzial einer solchen Art des Projektunterrichts in der Beschäftigung mit Neuem und Fremden besteht sowie darin, dass die daran Mitwirkenden sich auf Differenz- bzw. Kollisionserfahrungen einlassen (ausführlich siehe Pasuchin 2021b, 226ff.). Ein weiterer unerwarteter Aspekt war, dass im Rahmen dieses Projektzugangs (im Vergleich zu den anderen) eine besonders intensive Vermittlung von theoretischem Hintergrundwissen zur bearbeiteten Materie stattfand. Bei genauer Betrachtung stellte sich das erstens deswegen als weniger erstaunlich heraus, weil ungewöhnliche Erlebnisse Fragen evozieren, die nach Erklärung verlangen, und zweitens, da so ein Vorhaben aufgrund der starken Ausrichtung an Prozessen viele Räume dafür lässt, auf derartige Fragen einzugehen. Weniger geeignet erscheint der Ansatz hingegen im Hinblick auf die Umsetzung der Ideen der Beteiligten und hinsichtlich des Erwerbs praktischer Fertigkeiten. Erstere Aspekte fanden bei den Workshops von Luft zwar u. a. in den Phasen der Improvisation Berücksichtigung und letztere waren z. B. bei der Gestaltung von Musikstücken am Computer integriert; insgesamt spielten sie aber eine untergeordnete Rolle. Ebenso sind die Chancen der Reproduktion eines solchen Konzeptes durch andere Personen fraglich, weil die Lehrkräfte über grosses Spezialwissen auf einem eng eingegrenzten Gebiet verfügen und v. a. selbst eine enorme Begeisterung dafür mitbringen müssen, um es ‘authentisch) vermitteln zu können. Als gänzlich unwahrscheinlich erscheint vor dem Hintergrund der Erfahrungen aus der ersten Projektreihe die öffentliche Aufführung der Produkte und folglich auch ihre breite Rezeption.

Gerade im Ermöglichen derartiger Präsentationen und der damit verbundenen Aufmerksamkeit und Wertschätzung sowie im Evozieren des daraus resultierenden Stolzes der in die Arbeit involvierten Jugendlichen besteht das grösste Potenzial des mit (Kunst-)Werkorientierung benannten Zuganges. Da genau das zu den Hauptintentionen der Projekte der zweiten hier untersuchten Reihe gehörte und ihre Leiterin und ihr Leiter zwecks der Erreichung solcher Ziele enorme Ressourcen aufwendeten, war diese Beobachtung zu erwarten, ist also wenig erstaunlich. Eine Vorannahme, die sich jedoch keinesfalls bestätigte, bestand darin, dass die Vermittelnden - aufgrund ihrer eigenen Verortung im Kunstkontext und der Bemühung um die Positionierung der Produkte in diesem Feld - versuchen würden, ihre eigenen ästhetischen Vorstellungen zu verwirklichen bzw. den Teilnehmenden aufzuoktroyieren. Stattdessen waren sie durchgehend darauf bedacht, die Ideen der Schülerinnen und Schüler 
möglichst ‘originalgetreu umzusetzen. ${ }^{5}$ Dass ihnen das grösstenteils gelang, ist auch an der starken Anlehnung der Ergebnisse an jugendkulturellen Ausdrucksformen ersichtlich. Das hatte jedoch (durchaus zur Überraschung des Autors) zur Folge, dass die Schülerinnen und Schüler auf der ästhetischen Ebene bei diesen Projekten nur selten mit etwas konfrontiert wurden, was für sie tatsächlich neu oder sogar absolut fremd gewesen sein musste - auf jeden Fall ab den Phasen, die an die Einleitungen anschlossen. Abgesehen von den Anfangsetappen gab es auch kaum Zeit für die Behandlung von (theoretischen) Hintergrundinformationen, da danach sämtliche Bestrebungen darauf ausgerichtet waren, die Produkte in höchstmöglicher Qualität zu gestalten. Um das zu erreichen, wurden den Schülerinnen und Schülern einige praktische Fertigkeiten vermittelt, was jedoch selten auf einem Niveau stattfand, das sie dazu befähigt hätte, auch nur Teilergebnisse der jeweiligen Projekte vollkommen selbstständig fertigzustellen. ${ }^{6}$ Der unermessliche Zeitaufwand, den alle beteiligten Helferinnen und Helfer (abgesehen von Rešić und Stöllinger auch externe Fachleute, zum Schluss ebenso der Verfasser) in die Finalisierung der aufgeführten Produkte investierten, lässt die Wiederholung derartiger Vorhaben unter 〈regulären〉 Bedingungen eines Schulworkshops und erst recht eines gewöhnlichen Unterrichts illusorisch erscheinen.

Die Entwicklung von Konzepten für Projekte an den Schnittstellen zwischen Medien- und Kulturpädagogik, die über einmalige Ereignisse hinausgehen, und unter eher alltäglichen schulischen Rahmenbedingungen reproduzierbar bzw. auf andere Lehr-/Lernsituationen übertragbar sind, bildet eines der Hauptziele des dritten hier beschriebenen, als Vermittlungsorientierung bezeichneten Ansatzes. Die grösste Überraschung, die der Autor im Verlauf der darauf bezogenen Teilstudie erlebte, war ernüchternd. Vor dem Hintergrund jahrelanger Erfahrungen im Zuge der Begleitung von Studierenden seiner Lehrpraxiskurse beim (auf seinen Unterlagen basierenden) Unterricht an der Schule bestand die Erwartungshaltung darin, dass der Lehrende, der die Materialien für die Durchführung eines eigenen Projektes (ohne Unterstützung seitens des Verfassers) nutzte, damit problemlos zurechtkommen würde. Zwar waren die Umstände dieses Vorhabens bedingt durch die Corona-Situation alles andere als 〈regulär (Distanzunterricht, mehrere Unterbrechungen durch Erkrankungen etc.) und damit wenig (repräsentativ〉. Trotzdem kristallisierte sich daran heraus, dass die Umsetzung des Konzepts von Lehrpersonen entweder ein hohes Mass an technischer Expertise oder viel Zeit für die Vor- und Nachbereitungen verlangt

5 Das fiel dem Autor im Zuge der teilnehmenden (Selbst-)Beobachtung besonders auf, in deren Verlauf er sich darum bemühte, den Ansatz der Kollegin und des Kollegen zu imitieren und v. a. ihre Zielsetzung eines öffentlich aufführbaren Werkes zu übernehmen. Denn dabei kam er sich über weite Strecken wie ein Dienstleister vor, der die Vorgaben seiner Auftraggebenden (der Schülerinnen und Schüler) zu realisieren hatte.

6 Dazu, dass die Zielsetzung, Kinder und Jugendliche im Rahmen medien- und kulturpädagogischer Interventionen absolut selbstständig professionelle Produkte gestalten zu lassen, illusorisch und folglich wenig praxisrelevant ist, siehe Pasuchin 2022. 
- Faktoren, die nicht immer gegeben sind. Während sich das genannte Ziel insofern als nicht zur Gänze erreichbar herausstellte, erscheint jenes der Vermittlung von Fähigkeiten und Fertigkeiten auf der (eher) praktischen Ebene durchaus realistisch. Denn fast alle an den Projekten beteiligten Schülerinnen und Schüler sowie Studierenden konnten (zumeist nach anfänglichen Schwierigkeiten und anschliessenden Unterstützungen) im Verlauf der Arbeit sowohl die ästhetisch-formalen als auch die technischen Herausforderungen gut meistern und jene Abschnitte der Endprodukte, die sie gestalten sollten, grösstenteils selbstständig fertigstellen. Im Zuge dieser vermittlungsorientierten Projekte kamen manche der Mitwirkenden mit für sie fremden (u. a. als «krank» empfundenen) Ausdrucksformen in Berührung und bei der Kontextualisierung der Aufgaben wurden immer wieder auch Hintergrundinformationen behandelt. Jedoch bietet der erfahrungsorientierte Ansatz im Vergleich dazu offensichtlich viel grössere Chancen zur Auseinandersetzung mit Neuem und zur Erschliessung theoretischer Kenntnisse. Unter Einsatz der Methode des Autors konnten die Beteiligten einige ihrer Ideen (z. B. hinsichtlich der konkreten Projektthemen, ihrer eigenen Kurzperformances sowie der Gestaltung ihrer Strophen) umsetzen und erfahrungsgemäss ist es realistisch, mit den Ergebnissen solcher Vorhaben ein grösseres Publikum zu erreichen. Im Hinblick auf diese zwei Punkte scheint aber der (kunst-)werkorientierte Zugang bedeutend mehr Möglichkeiten zu bieten.

Das führt zur (wenig überraschenden) Gesamtschlussfolgerung, dass keine der hier behandelten Herangehensweisen zum medien-kulturpädagogischen Projektunterricht grundsätzlich «besser oder 'schlechter) ist als die andere. Vielmehr weisen sie unterschiedliche Potenziale im Hinblick auf divergierende Zielsetzungen auf, die folgenderweise veranschaulicht werden können:

\begin{tabular}{|c|c|c|c|}
\hline \multirow{2}{*}{ Potenziale einzelner Projektzugänge hinsichtlich... } & \multicolumn{3}{|c|}{ Orientierung an der/am } \\
\hline & Erfahrung & (Kunst-)Werk & Vermittlung \\
\hline \multirow{2}{*}{\multicolumn{4}{|c|}{$\begin{array}{l}\text {... Auseinandersetzung mit Neuem und Fremdem } \\
\text {... Erschliessung von theoretischen Kenntnissen }\end{array}$}} \\
\hline & & & \\
\hline \multicolumn{4}{|l|}{... öffentlicher Aufführung und breiter Rezeption } \\
\hline \multicolumn{4}{|l|}{... Umsetzung der Ideen der Beteiligten } \\
\hline ... Erwerbs von praktischen Fertigkeiten & & & \\
\hline ... Reproduzierbarkeit/Übertragbarkeit des Konzepts & & & \\
\hline
\end{tabular}

Tab. 1: Potenziale einzelner Projektzugänge.

\section{Fazit mit Fokus auf praktische Konsequenzen}

Die Konsequenzen, die aus allem Besprochenen im Hinblick auf die Konzeption medien-kulturpädagogischer Praxisprojekte gezogen werden können, hängen von zahlreichen Faktoren ab. Auf der interpersonalen ‘Mikroebene` zählen dazu einerseits 
die Persönlichkeiten der Leiterinnen und Leiter der Projekte sowie ihre (eng damit zusammenhängenden) spezifischen Vermittlungsmethoden und andererseits die Vorlieben sowie Dispositionen der Beteiligten, die einen starken Einfluss auf gruppendynamische Prozesse haben, die wiederum enorme Auswirkungen darauf aufweisen, wie die Lehrkräfte agieren (können). Auf der 〈Mesoebene` der unmittelbaren äusseren Rahmenbedingungen sind $u$. a. Facetten wie das Alter der Mitwirkenden, die Dauer und der Zeitpunkt der Abhaltung eines Projektes, die zur Verfügung stehende technische Ausstattung sowie die Zeitressourcen für die Vor- und Nachbereitung des Unterrichts zu berücksichtigen. Eine bedeutende Rolle spielen ausserdem Aspekte, die die «Makroebene` gesamtgesellschaftlicher Zusammenhänge betreffen - und hier vorrangig die soziale Schicht, der die Teilnehmerinnen und Teilnehmer der Massnahmen angehören (detailliert zu allen genannten Faktoren siehe Pasuchin 2021b, 198ff.). Insofern ist es - analog zu den zusammengefassten Erkenntnissen keinesfalls möglich, diese Konsequenzen zu (generalisieren) und erst recht nicht, sie in (Patentrezepte) umzuwandeln. Die einzige konkrete Empfehlung, die aus den Ausführungen resultiert, besteht darin, sich im Vorfeld eines Projektes sehr genau Gedanken darüber zu machen, was seine Zielsetzungen sind und ob bzw. in welcher Form sie unter den gegebenen Umständen realisierbar sein können.

Z. B. hat sich bei der Analyse des erfahrungsorientierten Zugangs von Luft herauskristallisiert, dass der Versuch seiner Verwirklichung lediglich Personen anzuraten ist, die nicht nur über sehr spezifisches Fachwissen und enorme Begeisterung für das jeweilige Thema verfügen, sondern auch einiges an (Frustrationstoleranz) vorzuweisen haben - d. h. sich nicht davon beirren lassen, wenn ihr Ansatz auf absolute Ablehnung stösst. Was man damit auf jeden Fall (gewinnt), ist die Chance, den Mitwirkenden neue Erlebnis- und Erkenntnisdimensionen zu eröffnen. Andererseits ist es offensichtlich innerhalb solcher Projekte kaum möglich, der (an medien- und kulturpädagogische Initiativen häufig gestellten) Anforderung der Generierung vorzeigbarer Ergebnisse zu entsprechen.

Hingegen scheint gerade in der Option der Herstellung von Produkten, die positive öffentliche Resonanz hervorrufen, der grosse Vorteil des (kunst-)werkorientierten Konzepts zu liegen. In Kombination mit dem Potenzial der Umsetzung von Ideen der Projektbeteiligten kann das durchaus ein anstrebenswertes Ziel von Vorhaben im hier behandelten Gebiet sein - v. a., wenn sie in einem sozioökonomisch belasteten bzw. belastenden Umfeld durchgeführt werden (Stichworte «Selbstwirksamkeit) und (Fremdwirksamkeit`). Bei der Überlegung, diesen Ansatz zu realisieren, ist jedoch zu bedenken, dass sich solche Erfolge - wie es an den Beobachtungen der Arbeit von Rešić und Stöllinger zu sehen war - erst auf Basis enormer zeitlicher Investitionen sowohl hinsichtlich der Projektdauer als auch (und vor allem) bezüglich des Vor- und Nachbereitungsaufwandes seitens der Leiterinnen und Leiter einstellen und folglich keinesfalls ‘vorprogrammiert sind. 
Was den Einsatz von Zeitressourcen betrifft, ist der vermittlungsorientierte Zugang zunächst nicht weniger aufwendig, da im Vorfeld derartiger Massnahmen ein detaillierter Ablaufplan zu erarbeiten ist und zahlreiche Unterrichtsmaterialien (Handouts, Musterbeispiele, Übungsdateien etc.) hergestellt werden müssen. Die Hoffnung auf die Reproduzierbarkeit solcher Modelle sowie die Wiederverwendung der Unterlagen (und damit auf zukünftige Zeit- und Energieersparnisse) ist zwar bis zu einem gewissen Grad gerechtfertigt, erfüllt sich jedoch (wie an den Projekten des Autors erkennbar wurde) nicht immer. Auf jeden Fall bewahrt es Lehrkräfte nicht davor, die eigenen Ansätze ständig weiterentwickeln und die Materialien anpassen zu müssen. Insofern erfordert auch diese Herangehensweise zu ihrer Umsetzung von den Leiterinnen und Leitern der Vorhaben spezifische Vorkenntnisse sowie Dispositionen. Zu den Letzteren gehört vorrangig die (wie sowohl bei Luft als auch bei Rešić und Stöllinger zu sehen war, bei Künstlerinnen und Künstlern nicht immer ausgeprägte) Freude an der Vermittlung praktischer ästhetisch-formaler und instrumenteller Fertigkeiten.

\section{Literatur}

Baacke, Dieter. 1999. «Medienkompetenz als zentrales Operationsfeld von Projekten». In Handbuch Medien: Medienkompetenz. Modelle und Projekte, herausgegeben von Dieter Baacke, Susanne Kornblum, Jürgen Lauffer, Lothar Mikos, und Günter A. Thiele, 31-35. Bonn: Bundeszentrale für politische Bildung.

Bourdieu, Pierre. 2014. Die feinen Unterschiede. Kritik der gesellschaftlichen Urteilskraft. 24. Auflage. Frankfurt a. M.: Suhrkamp.

Bundeszentrale für politische Bildung. o. J. «Datenbanken für kulturelle Projektarbeit». https://www.bpb.de/gesellschaft/bildung/kulturelle-bildung/59986/datenbanken.

DBR-Collective. 2003. «Design-Based Research: An Emerging Paradigm for Educational Inquiry». Educational Researcher 32 (1): 5-8. https://doi.org/10.3102/0013189X032001005.

Ellermann, Ulla und Flügge-Wollenberg, Barbara. 2013. «Tanz als Alltagskultur». KULTURELLE BILDUNG ONLINE. https://doi.org/10.25529/92552.226.

Euler, Dieter, und Peter F. E. Sloane. 2014. «Editorial». In Design-Based Research, herausgegeben von Dieter Euler und Peter F. E. Sloane, 5-11. Stuttgart: Franz Steiner.

Flick, Uwe. 2010. Triangulation. In Hauptbegriffe qualitativer Sozialforschung, hrsg. v. Ralf Bohnsack, Winfried Marotzki und Michael Meuser, 3. Auflage, 161-162. Köln: Böhlau.

Jahn, Dirk. 2014. «Durch das praktische Gestalten von didaktischen Designs nützliche Erkenntnisse gewinnen: Eine Einführung in die Gestaltungsforschung». Wirtschaft und Erziehung 66 (1): 3-15. https://www.fbzhl.fau.de/files/2014/06/artikel_jahn_wue_1-2014_sc1.pdf.

Lowinski, Felicitas. 2007. Bewegung im Dazwischen. Ein körperorientierter Ansatz für kulturpädagogische Projekte mit benachteiligten Jugendlichen. Bielefeld: transcript. https://doi. org/10.1515/9783839407264. 
Moser, Heinz. 2018. Praxisforschung - eine Forschungskonzeption mit Zukunft. In Forschungswerkstatt Medienpädagogik. Bd. 2. herausgegeben von Thomas Knaus, 449-78. München: kopead.

Pasuchin, Iwan. 2020. «Corona-Projekt». https://medialab.moz.ac.at/forschung/corona-projekt.

Pasuchin, Iwan. 2021a. KREATIVE KOLLISIONEN. Kontexte und Prozesse Kultureller Medienbildung. Eine pragmatistische Design-Based Research Studie. Bd. 1. München: kopaed. https:// doi.org/g3wd.

Pasuchin, Iwan. 2021b. KREATIVE KOLLISIONEN. Kontexte und Prozesse Kultureller Medienbildung. Eine pragmatistische Design-Based Research Studie. Bd. 2. München: kopaed. https:// doi.org/g3wf.

Pasuchin, Iwan. 2022. «Je professioneller das Produkt aussieht, desto weniger haben die Kinder selbst gemacht - Plädoyer für einen pragmatistischen Umgang mit kulturpädagogischen Dilemmata». https://www.kubi-online.de/artikel/je-professioneller-produkt-aussieht-desto-weniger-haben-kinder-selbst-gemacht-plaedoyer.

Reinmann, Gabi. 2005. «Innovation ohne Forschung? Ein Plädoyer für den Design-Based Research-Ansatz in der Lehr-Lernforschung». Unterrichtswissenschaft 33 (1): 52-69. https:// doi.org/10.25656/01:5787.

Reinmann, Gabi. 2019. «Die Selbstbezüglichkeit der hochschuldidaktischen Forschung und ihre Folgen für die Möglichkeiten des Erkennens». In Hochschulbildungsforschung: Theoretische, methodologische und methodische Denkanstöße für die Hochschuldidaktik, herausgegeben von Tobias Jenert, Gabi Reinmann, und Tobias Schmohl, 125-48. Berlin: Springer. https://doi.org/10.1007/978-3-658-20309-2_8.

Ruge, Wolfgang B. 2017. «Keine Disziplin in Schlumpfhausen. Anmerkungen zu einer Wissenschaftstheorie der Medienpädagogik». In Medienpädagogik. Eine Standortbestimmung, herausgegeben von Christine Trültzsch-Wijnen, 115-21. Baden-Baden: Nomos. https://doi. org/10.5771/9783845279718-13.

Schelga, Markus. 2005. «FW:1». In Kinder machen Kunst mit Medien, herausgegeben von Carmen Mörsch, und Nanna Lüth, 9-11. München: kopaed

Schell, Fred. 2008. «Projektorientierung». In Handbuch Medienpädagogik, herausgegeben von Uwe Sander, Friederike von Gross, und Kai-Uwe Hugger, 587-591. Wiesbaden: VS. https:// doi.org/10.1007/978-3-531-91158-8_86.

Strübing, Jörg. 2008. «Pragmatismus als epistemische Praxis. Der Beitrag der Grounded Theory zur Empirie-Theorie-Frage». In Zur Relevanz qualitativer Forschung, herausgegeben von Herbert Kalthoff, Stefan Hirschauer, und Gesa Lindemann, 279-311. Theoretische Empirie. Frankfurt a. M.: Suhrkamp.

Zeidler, Judith. 2009. «Projektarbeit». In Grundbegriffe Medienpädagogik - Praxis, herausgegeben von Bernd Schorb, Günther Anfang, und Kathrin Demmler, 245-74. München: kopaed. 